Objectives: To clarify how disease activity differs for each generation, especially in menopausal period.

Methods: Using the Japanese large RA cohort database (NinJa:National database of Rheumatic Diseases in Japan) of 2016, we divided 12257 RA females into three groups of age (under 44 years old $=1,45$ to 55 years old $=2$, defined as a menopausal group, over 56 years old=3) and analysed them cross-sectionally. We conducted a one-way ANOVA on disease activity indexes such as Tender joint count (TJC), Swollen joint count (SJC), DAS28, HAQ-DI.

Results: Table 1 shows the number of people per group, the duration of disease, the titer of RF/ACPA, and the proportion of drugs used. The average usage of prednisone and the use of biologics was the most common in group 1. In table 2, TJC was the largest in group $2(p<0.01)$. Furthermore, the difference between groups seen in TJC tends to be larger than TJC 28. There was no significant difference in SJC (SJC28) between three groups. Other disease activity indicators (ESR, CRP, DAS 28, HAQ-DI) were the largest in group $3(p<0.01)$ and the percentage of Boolean remission was also lowest in group $3(p=0.02)$.

Abstract AB0255 - Table 1. Characteristics of the three age groups

\begin{tabular}{lcccc}
\hline group & 1 & 2 & 3 & P-value \\
\hline Number of patients & 1071 & 1699 & 9487 & \\
Symptom duration, years & 8.2 & 10.5 & 15.4 & $\mathrm{p}<0.01$ \\
RF titer, (IU/mL) & 186 & 233 & 254 & $\mathrm{p}=0.06$ \\
ACPA titer, $(\mathrm{U} / \mathrm{mL})$ & 90 & 102 & 132 & $\mathrm{p}<0.01$ \\
On NSAIDs use, $\mathrm{n}(\%)$ & 392 & $710(41 \%)$ & 3668 & $\mathrm{p}<0.01$ \\
& $(36 \%)$ & & $(38 \%)$ & \\
Daily mean prednisone dose, $(\mathrm{mg} /$ & 4.31 & 3.95 & 3.86 & $\mathrm{p}=0.01$ \\
day) & & & & \\
On DMARDs, $\mathrm{n}(\%)$ & 984 & 1623 & 8835 & $\mathrm{p}<0.01$ \\
& $(91 \%)$ & $(95 \%)$ & $(93 \%)$ & \\
On a biologic, $\mathrm{n}(\%)$ & 435 & $550(32 \%)$ & 2570 & \\
& $(40 \%)$ & & $(27 \%)$ & \\
\hline
\end{tabular}

Abstract AB0255 - Table 2. Comparisons of values for disease outcomes of the three age groups

\begin{tabular}{lcccc}
\hline Tender joint count(TJC), mean & 1.65 & 2.17 & 1.97 & $\mathrm{p}<0.01$ \\
\hline Tender joint count(28 joints, TJC28), mean & 1.17 & 1.48 & 1.44 & $\mathrm{p}=0.01$ \\
Swollen joint count(SJC), mean & 1.35 & 1.49 & 1.52 & $\mathrm{p}=0.23$ \\
Swollen joint count(28 joints, SJC28), & 1.05 & 1.19 & 1.20 & $\mathrm{p}=0.12$ \\
mean & & & & \\
ESR, mean, (mm/h) & 17.3 & 18.8 & 29.7 & $\mathrm{p}<0.01$ \\
CRP, mean, (mg/dl) & 0.32 & 0.37 & 0.58 & $\mathrm{p}<0.01$ \\
DAS28, mean & 2.51 & 2.78 & 3.12 & $\mathrm{p}<0.01$ \\
HAQDI score, mean & 0.25 & 0.42 & 0.77 & $\mathrm{p}<0.01$ \\
Proportion in Boolean remission, $\mathrm{n}(\%)$ & 351 & 485 & 2145 & $\mathrm{p}=0.02$ \\
& $(32 \%)$ & $(28 \%)$ & $(22 \%)$ & \\
\hline
\end{tabular}

Conclusions: This study shows that TJC may increase during menopause, unlike other disease activity indicators. Especially in group 2, it is possible that the joints which can't be evaluated at TJC 28 are affecting the results because in TJC, compared with TJC 28, there is a larger group difference. Although further studies are needed, an increase in TJC may reflect menopausal joint symptoms.

REFERENCE:

[1] Pikwer M, et al. Ann Rheum Dis. 2012 Mar;71(3):378-81.

Disclosure of Interest: None declared

DOI: 10.1136/annrheumdis-2018-eular.5516

\section{AB0256 DIFFERENTIAL DIAGNOSIS OF SERONEGATIVE RA: CALCIUM PYROPHOSPHATE DIHYDRATE DEPOSITION DISEASE}

K. Paalanen ${ }^{1}$, K. Rannio ${ }^{2}$, T. Rannio ${ }^{1}$, J. Asikainen ${ }^{1}$, P. Hannonen ${ }^{1}$, T. Sokka ${ }^{1}$. ${ }^{1}$ Rheumatology; ${ }^{2}$ Radiology, Central Finland Central Hospital, Jyväskylä, Finland

Background: Calcium pyrophosphate deposition (CPPD) disease is caused by calcium pyrophosphate (CPP) crystals and seen mainly in elderly. Clinical presentation can be heterogeneous. The arthropathy of CPPD may mimic RA, particularly if involving common joints seen in RA. Diagnosis of CPPD arthritis is based on CPP crystals seen in synovial fluid (SF) analysis, chondrocalcinosis (CC) seen in radiographs and/or on typical clinical presentation for CPPD. Early diagnosis of CPPD can be challenging and a proportion of CPPD patients may be misdiagnosed. We demonstrate 17 cases with CPPD initially diagnosed and treated as seronegative RA.
Objectives: To increase awareness that CPPD disease may resemble seronegative RA and to characterise clinical and radiographic phenotypes of these CPPD patients.

Methods: Altogether 435 early seronegative early RA patients were clinically diagnosed in a single rheumatology centre and scheduled for 10 year follow-up. All clinical data and radiographs were collected and reviewed. Patients were rediagnosed as CPPD related arthritis if they had typical radiographic findings and suitable clinical pattern of CPPD or positive CPP crystal finding in SF. These patients are the subjects of this study.

Results: 17 patients were identified with a CPPD disease. The mean age at base line was 71.2 years, and $82 \%$ were women. In 7 (41.2\%) patients baseline symptoms were polyarticular, and in all these patients` wrist, MCP or PIP joints were affected; other symptomatic joints were hip (1 patient) and ankle (3 patients). The initial symptoms of 6 (35.3\%) patients were oligoarticular, including MCP and PIP joint involvement (2 patients) or wrist and MCP, PIP or MTP joint symptoms (4 patients). Four (23.5\%) patients were diagnosed as monoarthritis including ankle (1 patient) and wrist (3 patients). Seven patients (41.2\%) fulfilled 1987 ACR criteria for RA and the diagnosis of early RA of the other 10 patients $(58.8 \%)$ was based on clinical judgement. During the follow up period the SF analysis of 4 patients was available, 3 SFs showing positivity for CPP crystals. In 13 patients, SF had not been taken. In retrospect the baseline radiographs of 10 patients showed evidence of CC, either in wrists or knees. During follow up all patients developed typical clinical pattern for CPPD disease: chronic CPP crystal inflammatory arthritis (9 patients), acute CPP crystal arthritis (6 patients) and OA with CPPD (2 patients). All developed similar radiographic findings compatible with CPPD, including CC of triangular fibrocartilage (17 patients), CC of knee (9 patients), CC or narrowing of MCP joints (7 patients), CC of metatarsophalangeal (MTP) joints (4 patients), CC of symphysis pubis (1 patient), CC of glenohumeral joint (1 patient) and SLAC (5 patients). None of these patients developed typical RA-like erosions.

Conclusions: The prevalence of CPPD patients in our early seronegative RA patients was $3.9 \%$. CPPD disease can mimic seronegative RA at baseline and is important in the differential diagnosis of seronegative arthritis.

\section{REFERENCE:}

[1] Zhang W, Doherty $M$, Bardin $T$, et al. European league against rheumatism recommendations for calcium pyrophosphate deposition. Part I: Terminology and diagnosis. Ann Rheum Dis. 2011;70(4):563-570.

Disclosure of Interest: None declared

DOI: 10.1136/annrheumdis-2018-eular.3244

\section{AB0257 PHYSICAL ACTIVITY IN EARLY AND LONG-STANDING RA - RELATIONS TO DISEASE ACTIVITY, CARDIOVASCULAR RISK FACTORS AND ATHEROSCLEROSIS}

K.M. Hörnberg ${ }^{1}$, L. Ångström ${ }^{1}$, A. Södergren ${ }^{1}$, J. Pomeroy ${ }^{2}$, C. Sandberg ${ }^{1}$, B. Sundström ${ }^{1}$, S. Wăllberg-Jonsson ${ }^{1}{ }^{1}$ Department of Public Health and Clinical medicine/Rheumatolgy, Umeå, Sweden; ${ }^{2}$ Marshfield Clinic Research Institute, Marshfield, USA

Background: The excess risk for cardiovascular disease (CVD) in Rheumatoid Arthritis (RA), is partly attributable to traditional cardiovascular risk factors for CVD $^{1}$ and systemic inflammation, ${ }^{1,2}$ factors known to be modified by physical activity. 3,4

Objectives: The aim of this cross-sectional study was to objectively measure and compare the level of physical activity in patients with early and long-standing RA, and to analyse possible associations with disease activity, risk factors for CVD and measures of subclinical atherosclerosis.

Methods: This study included 84 patients with early and 37 with long-standing RA (disease duration, mean [SD] 1.4 [0.4] and 16.3 [2.3] years respectively). Physical activity was measured using a combined accelerometer and heart rate monitor and included total physical activity (counts/ $\mathrm{min}$ ), proportion of moderate to vigorous physical activity (MVPA) and sedentary time. Further assessments were; disease activity (ESR, DAS28), functional ability (HAQ), risk factors for CVD (blood lipids, i.e., triglycerides, high density lipoprotein [HDL], low density protein [LDL], blood glucose, blood pressure, waist circumference, body mass index [BMI]), body fat (Dual-energy X-ray), and early signs of atherosclerosis (pulse wave velocity $[\mathrm{PWV}]$, augmentation index $[\mathrm{Alx}]$ and carotid intima-media thickness [CIMT]).

Results: Physical activity variables did not differ between patients with early and long-standing RA. Thirty-seven $\%$ of the patients with early and $43 \%$ of the patients with long-standing RA did not reach WHOs recommended levels of MVPA. Univariate linear regression analyses with the two groups combined, showed associations between total physical activity and younger age, lower values for $\mathrm{HAQ}$ and disease activity (ESR), as well as more beneficial values 
for blood glucose, triglycerides, waist circumference, BMI, body fat, sleeping heart rate (SHR), systolic, diastolic and central blood pressure and pulse pressure, Aix, PWV, and cIMT. More time spent in MVPA was associated with younger age and with favourable values of blood glucose, HDL, LDL, waist circumference, SHR and PWV.

Abstract AB0257 - Table 1. Physical activity variables in patients with early and longstanding RA, presented as median with inter-quartile range (IQR). P-value refers to MannWhitney U-test.

\begin{tabular}{lccc}
\hline & $\begin{array}{c}\text { Early RA } \\
(\mathrm{n}=84)\end{array}$ & $\begin{array}{c}\text { Long-standing RA } \\
(\mathrm{n}=37)\end{array}$ & $\mathbf{p}$ \\
\hline $\begin{array}{l}\text { Accelerometry } \\
\text { (mean counts) } \\
\text { minute) }\end{array}$ & $\begin{array}{c}35.7 \\
(29.9)\end{array}$ & $38.1(24.3)$ & .915 \\
$\begin{array}{l}\text { MVPA } \\
\text { (\% of wear time) }\end{array}$ & $3.2(6.6)$ & $2.5(5.5)$ & .884 \\
$\begin{array}{l}\text { Sedentary time } \\
\text { (\% of wear time) }\end{array}$ & 53.9 & $52.0(13.3)$ & .328 \\
\hline
\end{tabular}

MVPA=Moderate to Vigorous Physical Activity=time $\geq 1.75$ xresting heart rate. Sedentary time=heart rate data with zero accelerometer counts.

Conclusions: Physical activity behaviour was similar in patients with early and long-standing RA. Total physical activity as well as more time spent in moderate to vigorous physical activity were associated with more favourable risk factors for CVD and measures of atherosclerosis. These results stress the importance of promoting physical activity in patients with $\mathrm{RA}$

\section{REFERENCES:}

[1] Crowson CS, et al. Annals of the rheumatic diseases 2018;77(1):48-54.

[2] Wallberg-Jonsson S, et al. The Journal of rheumatology 1999;26 (12):2562-71.

[3] Stavropoulos-Kalinoglou A, et al. Annals of the rheumatic diseases 2013;72(11):1819-25

[4] Metsios GS, et al. Annals of the rheumatic diseases 2014;73(4):748-51.

Disclosure of Interest: None declared

DOI: 10.1136/annrheumdis-2018-eular.3930

\section{AB0258 1 IMPACT OF CONTROLLING DISEASE ACTIVITY ON REGAINING NORMAL PHYSICAL FUNCTION, AND ACHIEVING NO OR LIMITED PAIN IN PATIENTS WITH RHEUMATOID ARTHRITIS TREATED WITH BARICITINIB}

K. de Vlam ${ }^{1}$, B. Fautrel ${ }^{2}$, M. van de Laar ${ }^{3}$, B.W. Kirkham ${ }^{4}$, R. Alten ${ }^{5}$, K V. Beneden ${ }^{6}$, F. de Leonardis ${ }^{7}$, P. Lopez-Romero ${ }^{8}$, C. Gaich $^{9}$, A. Quebe ${ }^{9}$, A. Cardoso ${ }^{9}$, R. Fleischmann ${ }^{10}$, P. Emery ${ }^{11}$. ${ }^{1}$ Department of Musculoskeletal Sciences, University Hospital Leuven, Leuven, Belgium; ${ }^{2}$ Department of Rheumatology, APHP, Pitié-Salpêtrière Hospital, Paris, France; ${ }^{3}$ Department of Rheumatology, Medisch Spectrum Twente, Enschede, Netherlands; ${ }^{4}$ Rheumatology Department, Guy's and St Thomas' NHS Foundation Trust, London, UK; ${ }^{5}$ Schlosspark Klinik, Berlin, Germany; ${ }^{6}$ Eli Lilly and Company, Brussels, Belgium; ${ }^{7}$ Eli Lilly and Company, Swiss, Switzerland; ${ }^{8}$ Eli Lilly and Company, Madrid, Spain; ${ }^{9}$ Eli Lilly and Company, Indianapolis; ${ }^{10}$ Metroplex Clinical Research Center, University of Texas Southwestern Medical Center, Dallas, USA; ${ }^{11}$ Leeds Institute of Rheumatic and Musculoskeletal Medicine, University of Leeds, Leeds Biomedical Research Centre, Leeds, UK

Background: Remission or low disease activity (LDA) are the recommended treatment (tx) targets in rheumatoid arthritis (RA). ${ }^{1}$ It is still unknown whether achieving remission/LDA is associated with normalisation of physical function, and limiting pain.

Objectives: To describe the impact of baricitinib (BARI) tx on regaining normal physical function, and achieving no/limited pain in patients (pts) who achieved remission or LDA, or remained in moderate or high disease activity (MDA, HDA). Methods: This is a post-hoc analysis of RA-BEAM (NCT01710358) and RABEGIN (NCT01711359). Mutually exclusive categories were defined as clinical disease activity index (CDAI) scores of $\leq 2.8$ (remission), $>2.8$ to $\leq 10$ (LDA), $>10$ to $\leq 22$ (MDA), and >22 (HDA). Last observation carried forward was used for pain visual analogue scale (0-100 mm VAS) and Health Assessment Questionnaire-Disability Index (HAQ-DI) to impute missing values. Descriptive analyses of the pts achieving normalisation of physical function was defined by a HAQ-DI score of $<0.5$ (normative value), and limited/no pain by pain VAS of $\leq 10 \mathrm{~mm}$ at week (wk) 12 and 24 as a function of disease activity.

Results: Overall, 1228 pts in RA-BEAM (448, PBO+MTX; 471, BARI +MTX; 309 ADA +MTX) and 543 pts in RA-BEGIN (190, MTX; 156, BARI; 197, BARI +MTX) were included. In RA-BEAM, among pts in remission at wk 12,\% pts achieving limited/no pain was numerically higher in BARI $(83 \% ; 33 / 40)$ group compared with ADA $(73 \% ; 16 / 22)$ and PBO $(67 \% ; 6 / 9)$; at wk 24, these percentages were $81 \%$ (61/75), 82\% (32/39), and 63\% (12/19) for BARI, ADA, and PBO, respectively. Among pts who achieved remission on BARI +MTX tx, normal physical function was reported in $65 \%(26 / 40)$ and $73 \%(55 / 75)$ of pts at wk 12 and 24 , respectively (Fig 1). For ADA +MTX treated pts, the proportion was $73 \%(16 / 22)$ at wk $12 \%$ and $69 \%(27 / 39)$ at wk 24. In RA-BEGIN, among pts in remission,\% pts with limited/no pain at wk 12 was numerically higher for BARI (96\%; 21/22) compared with BARI +MTX (82\%; 32/39) or MTX (64\%; 9/14); limited/no pain at wk 24 , was reported in $68 \%(23 / 34), 87 \%(40 / 46)$, and $77 \%(17 / 22)$ of pts treated with BARI, $\mathrm{BARI}+\mathrm{MTX}$, and MTX, respectively. Among pts in remission, $\%$ pts achieving normal HAQ-DI at wk 12 and 24 with BARI monotherapy were $91 \%(20 / 22)$ and $82 \%$ (28/34); BARI +MTX, 77\% (30/39) and 91\% (42/46); and MTX monotherapy, 79\% $(11 / 14)$ and $82 \%(18 / 22)$, respectively.

RA-BEAM*

RA-BEGIN

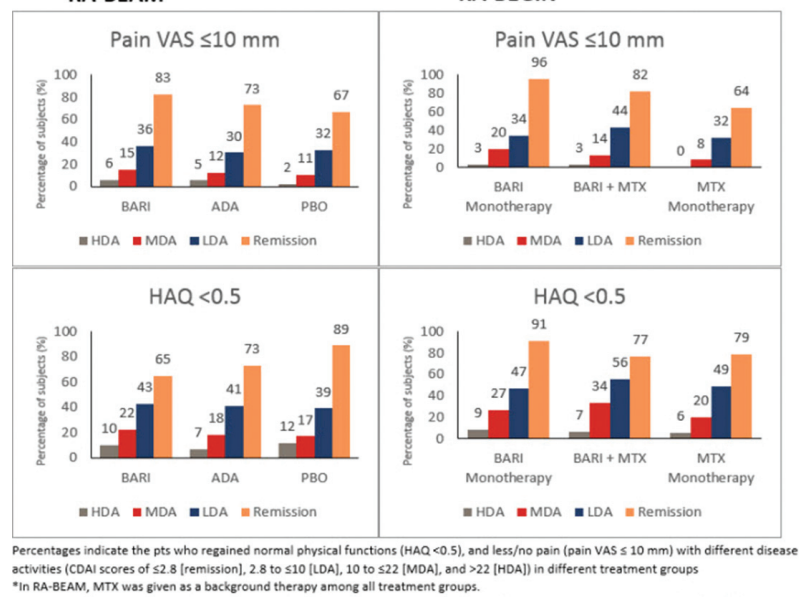

Abstract AB0258 - Figure 1. Percentage of pts who achieved pain VAS $\leq 10 \mathrm{~mm}$, and HAQ-DI $<0.5$ scores for the different disease activity states at wk 12

Percentages indicate the pts who regained normal physical functions $(H A Q<0.5)$ and less/no pain (pain VAS $\leq 10 \mathrm{~mm}$ ) with different disease activities (CDAI scores of $\leq 2.8$ [remission], $2.8 \leq 10$ [LDA], 10 to $\leq 22$ [MDA], and $>22$ [HDA]) in different treatment groups

*In RA-BEAM, MTX was given as a background therapy among all treatment groups.

Conclusions: These data support that controlling the disease activity by achieving remission or LDA increases the chances to regain normal physical function and relieve pain, independent of the $t x$. The data from RA-BEAM may indicate that achieving limited/no pain at wk 12 may be more likely with BARI vs ADA, when being in remission.

\section{REFERENCE:}

[1] Smolen JS, et al. Ann Rheum Dis. Jun 2017;76(6):960-977.

Disclosure of Interest: K. de Vlam Consultant for: Eli Lilly and Company, B. Fautrel Grant/research support from: AbbVie, MSD, Pfizer, Consultant for: AbbVie, Biogen, BMS, Celgene, Janssen, Lilly, Medac, MSD, NORDIC Pharma, Novartis, Pfizer, Roche, Sanofi-Aventis, SOBI, UCB, M. van de Laar Grant/ research support from: Abbvie, Janssen, Eli Lilly and Company, MSD, Pfizer, BMS, Consultant for: Janssen, Eli Lilly and Company, MSD, Pfizer, Sanofi, Novartis, B. Kirkham Grant/research support from: Arthritis Research UK, Abbvie, Eli Lilly and Company, Roche, UCB, Consultant for: Abbvie, Celgene, Eli Lilly and Company Janssen, Novartis, Pfizer, R. Alten Speakers bureau: Eli Lilly and Company, K. V. Beneden Employee of: Eli Lilly and Company, F. de Leonardis Employee of: Eli Lilly and Company, P. Lopez-Romero Employee of: Eli Lilly and Company, C. Gaich Shareholder of: Eli Lilly and Company, Employee of: Eli Lilly and Company, A. Quebe Shareholder of: Eli Lilly and Company, Employee of: El Lilly and Company, A. Cardoso Shareholder of: Eli Lilly and Company, Employee of: Eli Lilly and Company, R. Fleischmann Grant/research support from: AbbVie, Amgen, AstraZeneca, Bristol-Myers Squibb, Celgene, Genetech, GlaxoSmithKline, Janssen, Eli Lilly and Company, Merck, Pfizer, Regeneron, Roche, Sanofi, Aventis, UCB, Consultant for: AbbVie, Akros, Amgen, Bristol-Myers Squibb, 